\title{
Experimental Pseudomonas aeruginosa pneumonia: evaluation of the associated inflammatory response
}

\author{
O. Sibila*, C. Agustí*, A. Torres*, S. Baquero\#, S. Gando\#, J.R. Patrón ', J.G. Morato", \\ D.H. Goffredo ${ }^{\#}$, N. Bassi ${ }^{+}$and C.M. Luna ${ }^{\#}$
}

ABSTRACT: An abnormal inflammatory response (IR) in pneumonia is associated with poor outcomes and high mortality. Animal models could help to better understand the relationship between the pulmonary infection and the associated IR. The aims of the present study were to validate an experimental model of pneumonia induced by the inoculation of Pseudomonas aeruginosa in ventilated piglets and to study the associated IR over a long period of time (96 h).

Five Lagerwhite-Landrace piglets were ventilated for 4 days. After intubation, a solution containing $75 \mathrm{~mL}$ of $P$. aeruginosa $\left(10^{6}\right.$ colony-forming units $\left.\mathrm{mL}^{-1}\right)$ was bronchoscopically inoculated. Physiological and laboratory parameters were monitored throughout the study. Proinflammatory cytokines were measured in serum and in bronchoalveolar lavage (BAL). Histopathology of the lungs and cultures from blood, BAL and lungs were performed.

All the animals developed histopathological evidence of pneumonia. Microbiological studies of both BAL and lung confirmed the presence of $\boldsymbol{P}$. aeruginosa in all the samples. Throughout the study, an increase in interleukin-6 was observed in serum and in BAL.

In conclusion, the experimental model of pneumonia induced by the inoculation of high concentrations of Pseudomonas aeruginosa in ventilated piglets is feasible and could be appropriate for the evaluation of different aspects of the associated inflammatory response.

KEYWORDS: Experimental pneumonia, inflammatory response, interleukin-6, Pseudomonas aeruginosa, ventilated piglets

$\mathbf{P}$ atients with severe community- or hospital-acquired pneumonia requiring mechanical ventilation have a very high mortality [1, 2]. Over the last 10 yrs, evidence has been collected demonstrating the relevance of the host inflammatory response (IR) in the evolution of bacterial infections. Several studies in severe pneumonia $[3,4]$ have shown increased levels of different inflammatory cytokines both in the lung and in serum, and recent publications $[5,6]$ have demonstrated that an increased IR is a strong predictor of nonresponse to treatment and mortality in these patients. In clinical practice, different factors, such as the use of antibiotics or the presence of comorbidities, may influence the local and systemic IR, and the influence of these factors is difficult to assess accurately in humans. Animal models could help to clarify this issue. MARQUETTE et al. [7] have developed an animal model of pneumonia in mechanically ventilated piglets that closely resembles severe human pneumonia. This model has proved to be very useful for the proper evaluation of different aspects regarding diagnosis and treatment [8-13]. Pseudomonas aeruginosa is one of the most lethal causative microorganisms both in communityacquired pneumonia and ventilator-acquired pneumonia $[1,2,6]$. The incidence of $P$. aeruginosa pneumonia is increasing and involves patients that usually receive different antibiotics and suffer from diverse comorbidities and associated lung pathologies. These circumstances complicate the interpretation of the associated IR to this invasive microorganism. The availability of an animal model of severe pneumonia where potential confounders are excluded or controlled may improve the current knowledge on the intimal mechanisms involving the associated IR. This animal model has been used for pharmacological studies [13], including the evaluation of the effects of antibiotics [14]. In the present study, the clinical and histopathological consequences of $P$. aeruginosa inoculation were investigated, as well as the characteristics of the associated IR in animals ventilated for long periods of time (96 h).
AFFILIATIONS

*Pneumology Service, Thorax Clinic Institute, Barcelona Hospital ClinicIDIBAPS, University of Barcelona, Barcelona, Spain

\#Pneumology Division,

-University Centre of Experimental Surgery and

+Pathology Division, Hospital de Clínicas "José de San Martín", University of Buenos Aires, Buenos Aires, Argentina.

CORRESPONDENCE

A. Torres

Servei de Pneumologia

ICT

Hospital Clínic de Barcelona

Villarroel 170

Barcelona 08036

Spain

Fax: 34932275454

E-mail: atorres@ub.edu

Received:

May 042007

Accepted after revision:

August 222007

\section{SUPPORT STATEMENT}

This study was funded by grants from IDIBAPS, SOCAP, FUCAP, SEPAR, FIS PI 050136, FIS PI 030113 and CIBER CB 06/06/0028.

STATEMENT OF INTEREST

None declared.

European Respiratory Journal Print ISSN 0903-1936 Online ISSN 1399-3003 
It was hypothesised that inoculation of high concentrations of $P$. aeruginosa in ventilated piglets induces severe pneumonia. By doing so and by maintaining piglets ventilated for a long period of time, the histopathological derangement of lung parenchyma, as well as the associated IR, can be investigated. The aims of the present study were to validate an experimental model of pneumonia induced by the inoculation of $P$. aeruginosa in ventilated piglets and to assess the associated IR in the lung and in serum.

\section{METHODS}

An experimental intensive care unit was set up at the Hospital de Clínicas "José de San Martín", University of Buenos Aires, Buenos Aires, Argentina. It is fully equipped with cardiovascular monitors (model 78353-A; Hewlett-Packard, Andover, MA, USA), ventilators (Monal D; Air Liquide Santé, Paris, France) and electrical infusor pumps (Abbott Laboratories, Abbott Park, IL, USA).

\section{Animal preparation}

Six healthy domestic-bred Largewhite-Landrace piglets, aged 3 months and weighing $20 \pm 2 \mathrm{~kg}$ were anesthetised and orotracheally intubated with a 7.0-mm low-pressure cuff tube (Portex Ltd, Hythe, UK). They were pre-anaesthetised with intramuscular ketamine (Ketalar; Parke-Davis, Lansing, MI, USA) $250 \mathrm{mg}$. Anaesthesia was maintained with a continuous infusion of midazolam (Dormicum ${ }_{\mathbb{B}}$; Roche, Basel, Switzerland) $0.3 \mathrm{mg} \cdot \mathrm{kg}^{-1} \cdot \mathrm{h}^{-1}$, pancuronium bromide (Pavulon@ $4 \mathrm{mg}$; Organon, Buenos Aires, Argentina) $0.32 \mathrm{mg} \cdot \mathrm{kg}^{-1} \cdot \mathrm{h}^{-1}$ and phentanyl (Sublimaze $\subset 0.07 \mathrm{mg}$; Janssen-Cilag, Anvers, Belgium) $5 \mu \mathrm{g} \cdot \mathrm{kg}^{-1} \cdot \mathrm{h}^{-1}$. A catheter was inserted in the femoral vein for continuous infusion of $10 \%$ dextrose $\left(1.5 \mathrm{~mL} \cdot \mathrm{kg}^{-1} \cdot \mathrm{h}^{-1}\right)$ and Ringer lactate $\left(3 \mathrm{~mL} \cdot \mathrm{kg}^{-1} \cdot \mathrm{h}^{-1}\right)$ with an infusion pump. The femoral artery was cannulated with a $3 \mathrm{~F}$ polyethylene catheter (Plastimed, St Leu-la-Forêt, France) for pressure monitoring and blood sampling. An 8F suprapubic urinary catheter (Vesicoset; Angiomed, Karlsruhe, Germany) was placed in the bladder through surgical midline minipelvitomy. The piglets were then placed in a prone position and mechanically ventilated. Piglets that died during the first $12 \mathrm{~h}$ were excluded from the study.

\section{Mechanical ventilation}

Animals were mechanically ventilated in a volume-controlled mode. Ventilator parameters consisted of tidal volume $(V \mathrm{~T})$ at a constant inspiratory flow of $10 \mathrm{~mL} \cdot \mathrm{kg}^{-1}$, a respiratory frequency $(f \mathrm{R})$ of 15 breaths $\cdot \mathrm{min}^{-1}$, an inspiratory time of $33 \%$, with an initial inspiratory fraction $\left(\mathrm{FI}_{1} \mathrm{O}_{2}\right)$ of $100 \%$ and a positive end-expiratory pressure (PEEP) of 0 . Later, $\mathrm{FI}_{1} \mathrm{O}_{2}$ was set according to blood gas analysis in order to obtain arterial oxygen tension $\left(\mathrm{Pa}_{\mathrm{a}} \mathrm{O}_{2}\right) \geqslant 80$ and $<100 \mathrm{mmHg}$, and PEEP was increased to $\leqslant 5 \mathrm{cmH}_{2} 0$. Airway pressures, static lung compliance (calculated by dividing $V \mathrm{~T}$ by the difference between the end-inspiratory plateau pressure and the total PEEP [15]) and arterial blood gases (blood gas analyser IL-1306; Instrumentation Laboratories, Milan, Italy) were determined every $6 \mathrm{~h}$. Throughout the protocol, the carbon dioxide arterial tension was maintained at $35-45 \mathrm{mmHg}$ by increasing the $f \mathrm{R}$ to the maximum level preceding the appearance of auto-PEEP. Above this limit, hypercapnia was tolerated.

\section{Bronchial inoculation}

Animals were inoculated with $75 \mathrm{~mL}$ of a suspension of $10^{6}$ colony-forming units $(\mathrm{cfu}) \cdot \mathrm{mL}^{-1}$ of pathogenic $P$. aeruginosa ATCC 27853. Through the bronchoscope channel, $15 \mathrm{~mL}$ of the infusion was evenly distributed among every lobe of each lung.

The inocula of bacteria were instilled once the animals were haemodinamically stable after sedation and mechanical ventilation.

\section{Sampling and procedures}

Mechanical ventilation parameters $(V \mathrm{~T}, f \mathrm{R}$, airway pressures and $\mathrm{F} \mathrm{I}_{2} \mathrm{O}_{2}$ ), cardiac frequency, blood pressure, body temperature, blood gases, serum electrolytes (sodium, potassium) and lactate concentrations were monitored at $0,2,6,12,24,36,48$, $60,72,84$ and 96 h. Blood biochemistry (glucose, creatinine, urea, bilirrubin, aspartate aminotransferase and alanine aminotransferase) and blood cells count were obtained at 0, 24, 48, 72 and $96 \mathrm{~h}$.

\section{Bronchoalveolar lavage}

Five $20-\mathrm{mL}$ aliquots of sterile saline solution $(0.9 \%$ sodium chloride) were instilled and re-aspirated through the bronchoscope's channel in the right middle lobe at $0 \mathrm{~h}$ (before the inoculation of the $P$. aeruginosa suspension) and at $96 \mathrm{~h}$.

\section{Inflammatory parameters}

C-reactive protein

C-reactive protein (CRP) was quantified in serum and bronchoalveolar lavage (BAL) using a CRP kit (Biosystems S.A., Barcelona, Spain).

\section{Cytokines in blood and BAL fluid}

Tumour necrosis factor (TNF)- $\alpha$ and interleukin (IL)-1 $\beta,-6$ and -8 levels were measured in serum and BAL supernatant using the ELISA method in specific porcine kits (R\&D Systems Inc., Minneapolis, MN, USA).

BAL cytokines and CRP were determined at the time of intubation and at the end of the study. Serum cytokines and CRP were determined at the time of intubation and at $24,48,72$ and $96 \mathrm{~h}$.

\section{Bacteriological studies}

Quantitative cultures of BAL fluid, serum and lung tissue were performed at the end of the study.

BAL and lung tissue specimens were processed for quantitative bacterial cultures as described elsewhere [16], according to recommended laboratory methods [17].

\section{Sacrifice and post mortem studies}

Sacrifice was performed at $96 \mathrm{~h}$ under general anaesthesia by intravenous potassium chloride infusion.

\section{Collection of lung specimens}

After death, the animals remained under mechanical ventilation until surgical samples of lung parenchyma were obtained for bacteriological and histopathological evaluation. The lungs were aseptically exposed through a cervicothoracic midline incision. Thereafter, at least one lung tissue specimen $\left(1 \mathrm{~cm}^{3}\right)$ was taken from both the more preserved lobe (macroscopically) and the more involved lobe. 


\section{Histopathological assessment}

Lung tissue was processed according to standard methods. Analysis of vessels (thrombosis and endothelial lesions) and pleural (acute or chronic pleuritis) and lung parenchyma was performed. The evaluation of lung parenchyma included severity of pneumonia and presence of other associated lesions (hyaline membranes and alveolar damage). Severity of pneumonia was graded according to previously published criteria [18] as follows: $0=$ no pneumonia; $1=$ purulent mucous plugging; 2 =bronchiolitis; 3 =pneumonia (consolidation coexisting with significant accumulation of polymorphonuclear leukocytes, fibrinous exudates and cellular debris into the alveolar space); $4=$ confluent pneumonia (extension along different secondary lobes); and $5=$ abscessed pneumonia (cellular necrosis coexisting with disruption of cellular architecture). Pneumonia was limited to the last three categories. Classification of each specimen was based upon the worst category observed.

\section{Statistical analysis}

All data were expressed as mean \pm SD or SEM as appropriate. Nonparametric tests for paired data were used; Wilcoxon and Friedman tests were used for comparison of two or more than two time-points, respectively. A p-value $<0.05$ was considered statistically significant.

\section{Approval by the institutional committee}

All animals were treated in compliance with the guidelines of the Ethics Committee and Direction of Investigation of the Hospital de Clínicas "José de San Martín" and the "Guide for the Care and Use of Laboratory Animals" [19].

\section{RESULTS}

A total of five piglets were studied after excluding one that died during the first $12 \mathrm{~h}$ (death attributable to the animal preparation).

\section{Physiological and laboratory data}

Physiological and laboratory variables are shown in table 1. After bronchial inoculation, a rapid and persistent decrease of the $\mathrm{Pa}, \mathrm{O}_{2} / \mathrm{FI}_{1} \mathrm{O}_{2}$ ratio $(\mathrm{p}=0.01)$ and a rise in body temperature $(p=0.03)$ were observed throughout the study. Static lung compliance decreased over time, although differences did not reach statistical significance. No differences in biochemical data were observed.

\section{Inflammatory response}

Serum IL-6 increased throughout the study, with a maximal increase at $48 \mathrm{~h}$. IL-1 $\beta$ and -8 did not change significantly. Although not significant, levels of TNF- $\alpha$ decreased slightly at the end of the study (fig. 1).

A significant increase in the concentration of IL-6 in BAL fluid at the end of the study was also observed. The increase in the concentration of the remaining cytokines evaluated did not reach statistical significance (fig. 2). Levels of CRP in BAL fluid did not change over the study period (data not shown).

\section{Microbiology}

$P$. aeruginosa was present in all samples from BAL fluid and lung tissue cultures from the five piglets evaluated. BAL cultures yielded $P$. aeruginosa in a concentration $>10^{4} \mathrm{cfu} \cdot \mathrm{mL}^{-1}$ in four piglets and $>10^{5} \mathrm{cfu} \cdot \mathrm{mL}^{-1}$ in one piglet. Lung tissue cultures also showed growth of $P$. aeruginosa in a concentration

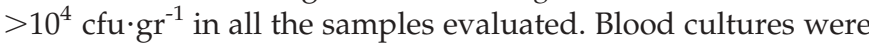
positive for $P$. aeruginosa in one piglet.

Morganella morgani and Staphylococcus coagulase-negative (both in a concentration of $10^{3} \mathrm{cfu} \cdot \mathrm{mL}^{-1}$ ) were also detected in two and three samples out of 20 evaluated, respectively.

\section{Histopathological findings}

All piglets developed pneumonia (grades 3-5). Pneumonia was present in all the samples taken from the most involved lobes and in three out of the five samples taken from the most

TABLE 1 Sequential measurements of physiological and laboratory parameters

\begin{tabular}{|c|c|c|c|c|c|c|}
\hline & $\mathrm{Oh}$ & $24 \mathrm{~h}$ & $48 \mathrm{~h}$ & $72 \mathrm{~h}$ & $96 \mathrm{~h}$ & p-value ${ }^{\#}$ \\
\hline Temperature ${ }^{\circ}$ & $36.4 \pm 0.7$ & $40.1 \pm 0.7$ & $39.0 \pm 1.2$ & $39.0 \pm 1.7$ & $39.1 \pm 1.7$ & 0.032 \\
\hline Cardiac frequency bpm & $146 \pm 36$ & $156 \pm 39$ & $138 \pm 11$ & $141 \pm 20$ & $140 \pm 29$ & 0.35 \\
\hline Mean $\mathrm{Pa}, \mathrm{O}_{2} \mathrm{mmHg}$ & $124 \pm 4$ & $114 \pm 19$ & $123 \pm 22$ & $118 \pm 21$ & $108 \pm 27$ & 0.58 \\
\hline $\mathrm{CL}, \mathrm{s} \mathrm{mL} \cdot \mathrm{cmH}_{2} \mathrm{O}^{-1}$ & $16.9 \pm 3.1$ & $16.4 \pm 3.2$ & $14.8 \pm 3.9$ & $13.8 \pm 4.0$ & $13.2 \pm 3.2$ & 0.14 \\
\hline $\mathrm{Pa}, \mathrm{O}_{2} / \mathrm{Fl}, \mathrm{O}_{2} \mathrm{mmHg}$ & $350 \pm 144$ & $210 \pm 98$ & $150 \pm 52$ & $138 \pm 50$ & $132 \pm 41$ & 0.017 \\
\hline Haematocrit $L \cdot L^{-1}$ & $30 \pm 1$ & $29 \pm 2$ & $27 \pm 3$ & $25 \pm 1$ & $22 \pm 1$ & 0.06 \\
\hline WBC count $\times 10^{9}$ cells $\cdot L^{-1}$ & $18160 \pm 10046$ & $21920 \pm 12934$ & $13160 \pm 3922$ & $10820 \pm 5140$ & $12340 \pm 4160$ & 0.09 \\
\hline Creatinine $\mathrm{mg} \cdot \mathrm{dL}^{-1}$ & $0.6 \pm 0.1$ & $1.1 \pm 0.8$ & $0.6 \pm 0.3$ & $0.4 \pm 0.1$ & $0.4 \pm 0.1$ & 0.52 \\
\hline Bilirubin $\mathrm{mg} \cdot \mathrm{dL}^{-1}$ & $0.4 \pm 0.1$ & $0.3 \pm 0.2$ & $0.3 \pm 0.2$ & $0.3 \pm 0.1$ & $0.3 \pm 0.1$ & 0.83 \\
\hline ASAT $\mathbf{U} \cdot \mathrm{dL}^{-1}$ & $61 \pm 46$ & $177 \pm 285$ & $255 \pm 229$ & $192 \pm 269$ & $130 \pm 146$ & 0.78 \\
\hline ALAT $\mathrm{U} \cdot \mathrm{dL}^{-1}$ & $46 \pm 15$ & $64 \pm 42$ & $69 \pm 56$ & $79 \pm 81$ & $70 \pm 62$ & 0.99 \\
\hline CRP $\mathrm{mg} \cdot \mathrm{dL}^{-1}$ & $2.9 \pm 1.7$ & $4.9 \pm 3.9$ & $5.5 \pm 3.5$ & $3.7 \pm 0.9$ & $3.6 \pm 2.2$ & 0.71 \\
\hline
\end{tabular}

Data are presented as mean $\pm \mathrm{SD}$, unless otherwise stated. bpm: beats per minute; $\mathrm{Pa}_{2} \mathrm{O}_{2}$ : arterial oxygen tension; $\mathrm{CL}, \mathrm{s}$ : static lung compliance; $\mathrm{Fl}, \mathrm{O}_{2}$ : inspiratory fraction WBC: white blood cell; ASAT: aspartate amine transferase; ALAT: alanine amine transferase; CRP: C-reactive protein. *: calculated according to the Friedman test. $1 \mathrm{mmHg}=0.133 \mathrm{kPa}$. 

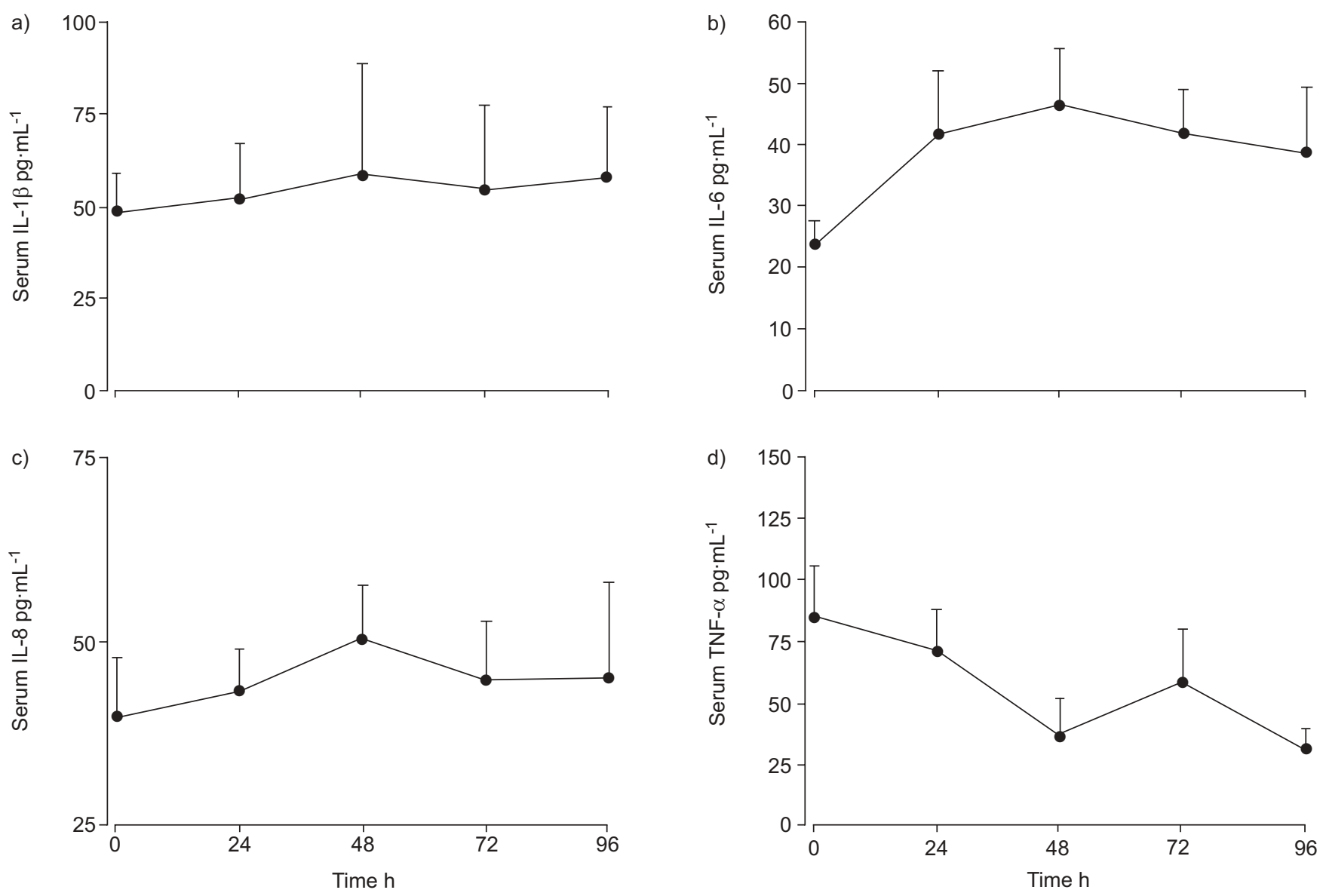

FIGURE 1. Sequential determination of serum a) interleukin (IL)-1 $1 \beta$, b) IL-6, c) IL-8 and d) tumour necrosis factor (TNF)- $\alpha$ during the study. Data are presented as mean \pm SEM. a) $p=0.97, b) p=0.04$, c) $p=0.67$ and d) $p=0.30$ (calculated according to Friedman test).

preserved lobes. Severe pneumonia, defined as abscessed pneumonia or confluent pneumonia (grades 4 and 5), was present in $60 \%$ of the pulmonary biopsies and in three out of five piglets (table 2). Acute or subacute pleuritis was also present in all the piglets; diffuse alveolar damage, defined by the presence of hyaline membranes covering the alveolar epithelium was present in four out of five piglets; and vascular lesions, including endothelial or thrombosis, was present in three out of five.

\section{DISCUSSION}

The results of the present study demonstrate that inoculation of $P$. aeruginosa into the lungs in ventilated piglets causes pneumonia. After a long period of mechanical ventilation $(96 \mathrm{~h})$ all the animals developed pneumonia by this microorganism and severe histopathological lesions in the lungs were observed in the majority of piglets. In addition, using this animal model, the present authors were able to evaluate the dynamics of the associated IR, which was characterised by a significant increase in IL-6 levels over time in both serum and BAL.

The availability of an experimental model of severe pneumonia, in which animals are ventilated during $96 \mathrm{~h}$ without the interference of antibiotic treatment, allows the physiopathological changes taking place in the lung to be studied throughout the process. Inoculation of $P$. aeruginosa led to the development of clinical signs of pneumonia (fever and gas exchange impairment) and deterioration in lung mechanics (decrease in static compliance over time). Post mortem evaluation confirmed both histopathologically and microbiologically the presence of pneumonia in all the animals evaluated.

The presence of pneumonia was evident in all the lobes that macroscopically were most involved but also in $60 \%$ of the most preserved ones, confirming the multilobar distribution of the infection. Interestingly, there was also evidence of hyaline membranes in $80 \%$ of the piglets evaluated. Hyaline membranes appear later in the evolution of pneumonia and their presence is associated with diffuse alveolar damage and irreversible fibrosis [20]. GOLDSTEIN et al. [9] evaluated lesions associated with acute pneumonia in the same animal model and, as expected, no hyaline membranes or signs of diffuse alveolar damage were observed. By contrast, in the present study, the development of late-stage lesions of pneumonia was common, as animals were ventilated for long periods of time. This circumstance might be useful for the assessment of different types of treatment that have been claimed to be beneficial in these late stages, such as glucocorticoids or other immunomodulators. 

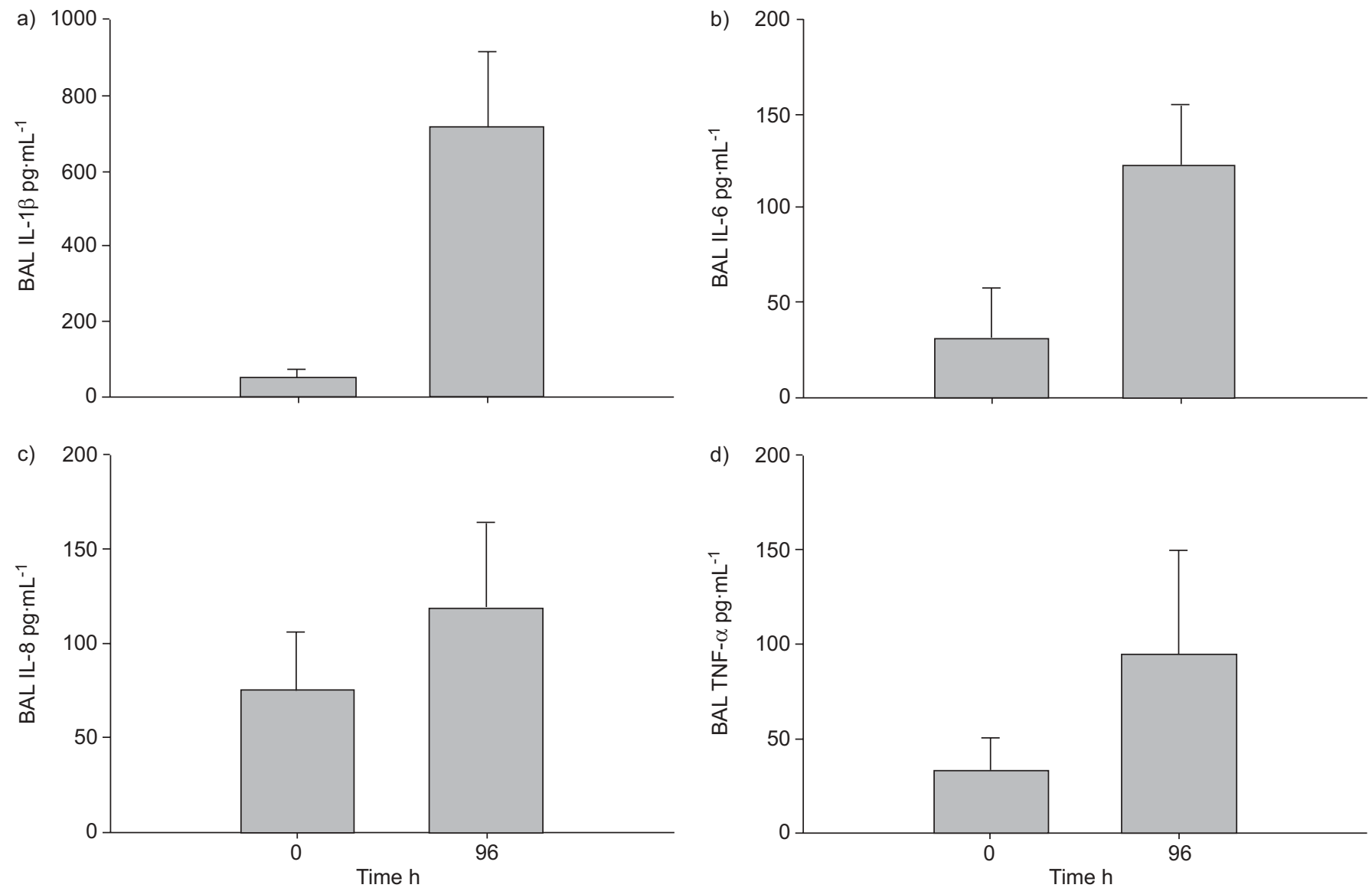

FIGURE 2. Bronchoalveolar lavage (BAL) measurements at 0 and $96 \mathrm{~h}$ of the initiation of the study of a) interleukin (IL)-1 $1 \beta$, b) IL-6, c) IL-8 and d) tumour necrosis factor (TNF)- $\alpha$. Data are presented as mean \pm SEM. a) $p=0.06$, b) $p=0.04$, c) $p=0.14$ and d) $p=0.46$ (calculated according to Wilcoxon test).

In the present study, $P$. aeruginosa was selected as the aetiological agent, as it is a common and often lethal cause of pneumonia in ventilated patients [2]. Patients with $P$. aeruginosa pneumonia usually receive different types of antibiotics and suffer from several comorbidities, circumstances that may influence the associated IR. By using this animal model of pneumonia, some of the potential confounders were avoided, making the assessment of the IR more accurate.

The lung IR at the end of the study was characterised by an increase in different pro-inflammatory cytokines, mainly IL-6,

\section{TABLE 2 Histopathological findings}

\begin{tabular}{lcc} 
Piglet & \multicolumn{2}{c}{ Pneumonia score } \\
\cline { 2 - 3 } & More involved lobe & More preserved lobe \\
\hline $\mathbf{1}$ & Abscessed pneumonia & Confluent pneumonia \\
$\mathbf{2}$ & Confluent pneumonia & Confluent pneumonia \\
$\mathbf{3}$ & Pneumonia & Purulent mucus plugging \\
$\mathbf{4}$ & Abscessed pneumonia & Confluent pneumonia \\
$\mathbf{5}$ & Pneumonia & Purulent mucus plugging \\
\hline
\end{tabular}

in BAL fluid. Although an increase in IL-6 levels was also observed in serum, the dynamics of the remaining cytokines evaluated in blood did not show a consistent pattern, suggesting that the IR was mainly compartmentalised. DeHOux et al. [21] confirmed that the production of cytokines in response to local bacterial infection is compartimentalised in a study in humans with unilateral pneumonia. However, when the pneumonia is more severe, causes septic shock or is acquired during mechanical ventilation, the systemic IR is also evident $[3,4,22]$. The lack of correlation between local and systemic inflammation and the inconsistent pattern of the different cytokines measured in serum may probably be explained by the absence of sepsis in the animals studied, as reflected by negative blood cultures in all but one animal, no haemodynamic disturbances and no requirement for vasopressive drugs. In the present study, IL- 6 was the only of the cytokines evaluated that showed a significant increase (both in serum and in the lung). In fact, correlations between severity scores and serum IL-6 have been observed [23, 24]. In addition, IL-6 concentration in serum has been shown to be an independent predictive factor of mortality in different population groups [5, 6]. In this sense, measurement of IL-6 concentration in serum and in BAL might be a very useful parameter to assess the magnitude of the IR and the potential effects of different anti-inflammatory treatments. 
The present study has limitations. First, the exogenous administration of a highly bacterial inoculum in a previously healthy animal does not necessarily reflect the complexities of pneumonia development in humans. Potential species differences in lung immunology between piglets and humans must also be considered. Also, sequential BAL samples at different time-points would have been optimal for an accurate evaluation of the local IR. Although the "early-phase" cytokines evaluated in the present study have been widely used in clinical practice for the assessment of the IR, other inflammatory mediators, such as adhesion proteins, regulated on activation, normal T-cell expressed and secreted, or IL-10, might have been more appropriate, as they are expressed in more advanced phases of the infection. Finally, the fact that the injury of mechanical ventilation might have played some role in the observed IR cannot be excluded.

In summary, the present animal model of pneumonia by Pseudomonas aeruginosa has proved to be efficacious and reproducible, and may constitute a useful tool for evaluating the inflammatory response associated with pulmonary infections and the potential effects of immunomodulatory treatments.

\section{REFERENCES}

1 Bodí M, Rodríguez A, Solé-Violán J, et al. Antibiotic prescription for community-acquired pneumonia in the intensive care unit: impact of adherence to Infectious Diseases Society of America guidelines on survival. Clin Infect Dis 2005; 41: 1709-1716.

2 Chastre J, Fagon JY. Ventilator-associated pneumonia. Am J Respir Crit Care Med 2002; 165: 867-903.

3 Montón C, Torres A, El-Ebiary M, Filella X, Xaubet A, de la Bellacasa JP. Cytokine expression in severe pneumonia: a bronchoalveolar lavage study. Crit Care Med 1999; 27: 1745-1753.

4 Schütte H, Lohmeyer J, Rosseau S, et al. Bronchoalveolar and systemic cytokine profiles in patients with ARDS, severe pneumonia and cardiogenic pulmonary oedema. Eur Respir J 1996; 9: 1858-1867.

5 Fernández-Serrano S, Dorca J, Coromines M, Carratalá J, Gudiol F, Manresa F. Molecular inflammatory responses measured in blood of patients with severe communityacquired pneumonia. Clin Diagn Lab Immunol 2003; 10: 813-820.

6 Ioanas M, Ferrer M, Cavalcanti M, et al. Causes and predictors of nonresponse to treatment of the intensive care unit-acquired pneumonia. Crit Care Med 2004; 32: 938-945.

7 Marquette $\mathrm{CH}$, Wermert D, Wallet F, Copin MC, Tonnel AB. Characterization of an animal model of ventilator-acquired pneumonia. Chest 1999; 115: 200-209.

8 Wermert D, Marquette CH, Copin MC, et al. Influence of pulmonary bacteriology and histology on the yield of diagnostic procedures in ventilator-acquired pneumonia. Am J Respir Crit Care Med 1998; 158: 139-147.

9 Goldstein I, Bughalo MT, Marquette $\mathrm{CH}$, et al. Mechanical ventilation-induced air-space enlargement during experimental pneumonia in piglets. Am J Respir Crit Care Med 2001; 163: 958-964.

10 Goldstein I, Wallet F, Nicolas-Robin A, Ferrari F, Marquette $\mathrm{CH}$, Rouby JJ. Lung deposition and efficiency of nebulized amikacin during Escherichia coli pneumonia in ventilated piglets. Am J Respir Crit Care Med 2002; 166: 1375-1381.

11 Elman M, Goldstein I, Marquette $\mathrm{CH}$, et al. Influence of lung aeration on pulmonary concentrations of nebulized and intravenous amikacin in ventilated piglets with severe bronchopneumonia. Anesthesiology 2002; 97: 199-206.

12 Ferrari F, Goldstein I, Nieszkowszka A, et al. Lack of lung tissue and systemic accumulation after consecutive daily aerosols of amikacin in ventilated piglets with healthy lungs. Anesthesiology 2003; 98: 1016-1019.

13 Tonnellier M, Ferrari F, Goldstein I, Sartorius A, Marquette $\mathrm{CH}$, Rouby JJ. Intravenous versus nebulized ceftazidime in ventilated piglets with and without experimental bronchopneumonia: comparative effects of helium and nitrogen. Anesthesiology 2005; 102: 995-1000.

14 Luna CM, Baquero S, Gando S, et al. Experimental severe Pseudomonas aeruginosa pneumonia and antibiotic therapy in piglets receiving mechanical ventilation. Chest 2007; 132: 523-531.

15 Rossi A, Polese G, Brandi G, Conti G. Intrinsic positive end-expiratory pressure (PEEPi). Intensive Care Med 1995; 21: 522-536.

16 El-Ebiary M, Torres A, González J, et al. Quantitative cultures of endotracheal aspirates for the diagnosis of ventilator-associated pneumonia. Am Rev Respir Dis 1993; 148: 1552-1557.

17 Balows A, Hausler WJJ, eds. Manual of Clinical Microbiology. 5th Edn. Washington, American Society for Microbiology, 1991.

18 Marquette $\mathrm{CH}$, Copin MC, Wallet F, et al. Diagnostic tests for pneumonia in ventilated patients: prospective evaluation of diagnostic accuracy using histology as a diagnostic gold standard. Am J Respir Crit Care Med 1995; 151: 1878-1888.

19 US Department of Health and Human Services. Guide for the Care and Use of Laboratory Animals. National Institute of Health Publication No. 93-23, revised 1985.

20 Meduri GU, Eltorky M, Winer-Muram HT. The fibroproliferative phase of late adult respiratory distress syndrome. Semin Respir Infect 1995; 10: 154-175.

21 Dehoux MS, Boutten A, Ostinelli J, et al. Compartmentalized cytokine production within the human lung in unilateral pneumonia. Am J Respir Crit Care Med 1994; 150: 710-716.

22 Bauer TT, Montón C, Torres A, et al. Comparison of systemic cytokine levels in patients with acute respiratory distress syndrome, severe pneumonia, and controls. Thorax 2000; 55: 46-52.

23 Glynn P, Coakley R, Kilgallen I, Murphy N, O’Neill S. Circulating interleukin 6 and interleukin 10 in community acquired pneumonia. Thorax 1999; 54: 51-55.

24 Antunes G, Evans SA, Lordan JL, Frew AJ. Systemic cytokine levels in community-acquired pneumonia and their association with disease severity. Eur Respir J 2002; 20: 990-995. 